\title{
MODELLING OF NATURAL CONVECTION FLOWS WITH LARGE TEMPERATURE DIFFERENCES: A BENCHMARK PROBLEM FOR LOW MACH NUMBER SOLVERS. PART 2. CONTRIBUTIONS TO THE JUNE 2004 CONFERENCE
}

\author{
Henri Paillère ${ }^{1}$, Patrick Le Quéré ${ }^{2}$, Catherine Weisman ${ }^{2}$, Jan Vierendeels ${ }^{3}$, \\ Erik Dick ${ }^{3}$, Malte Braack ${ }^{4}$, Frédéric Dabbene ${ }^{1}$, Alberto Beccantini ${ }^{1}$, \\ Etienne Studer ${ }^{1}$, Thibaud Kloczko ${ }^{1}$, Christophe Corre $^{5}$, Vincent Heuveline ${ }^{4}$, \\ Masoud Darbandi ${ }^{6}$ And SEyed FARID HosseinizadeH ${ }^{6}$
}

\begin{abstract}
In the second part of the paper, we compare the solutions produced in the framework of the conference "Mathematical and numerical aspects of low Mach number flows" organized by INRIA and MAB in Porquerolles, June 2004, to the reference solutions described in Part 1. We make some recommendations on how to produce good quality solutions, and list a number of pitfalls to be avoided.
\end{abstract}

Mathematics Subject Classification. 65M50, 76M10, 76M12, 76M20, 76M22, 76R10.

Numerical workshop, Low Mach Number Flows Conference, June 21-25, 2004, Porquerolles, France.

\section{INTRODUCTION}

The description of the benchmark problem is given in Part 1. of the paper and is not repeated here. The specifications of the benchmark with the required output format were detailed on the web-site of the conference, six months before the conference took place. The participants were asked to produce grid-converged results, or to ensure that their numerical results were sufficiently accurate by refining the mesh until the solutions varied no more (at least for the first four digits).

\footnotetext{
Keywords and phrases. Natural convection, non-Boussinesq, low Mach number.

1 CEA Saclay, DEN/DM2S/SFME,91191 Gif-sur-Yvette Cedex, France. henri.paillere@cea.fr; frederic.dabbene@cea.fr; beccantini@cea.fr; estuder@cea.fr; thibaud.kloczko@cea.fr

2 LIMSI, BP 133, 91403 Orsay Cedex, France. plq@limsi.fr; weisman@limsi.fr

3 Ghent University, 9000 Gent, Belgium. Jan.Vierendeels@rug.ac.be; erik.dick@UGent.be

${ }^{4}$ Heidelberg University, Germany. malte.braack@iwr.uni-heidelberg.de; vincent.heuveline@iwr.uni-heidelberg.de

5 SINUMEF Lab., ENSAM, 151 bd. de l'Hôpital, 75013 Paris, France. corre@paris.ensam.fr

6 Sharif University of Technology, Tehran, Iran. darbandi@sharif .edu
} 


\section{Methods And CONTRIBUtors}

\subsection{J. Vierendeels and E. Dick, Ghent University, Belgium}

This participant contributed to the reference solution in 2000, but repeated the calculations on even finer meshes. Solutions of the full compressible Navier-Stokes equations are computed using an explicit third-order discretization for the convective part and a line-implicit central discretization for the acoustic and the diffusive parts [7]. Multigrid technique provides convergence acceleration and eventually convergence behaviour is independent of grid size, grid aspect ratio, Mach number and Rayleigh number.

\subsection{Braack, Heidelberg University, Germany}

This participant also contributed to the reference solution, and repeated the calculations using a more accurate method (quadratic Finite Elements). A Finite Element discretization is used to solve the full compressible Navier-Stokes equations. The Finite Element space consists of equal-order quadratic ansatz functions. The stability is achieved by local projections of small-scale fluctuations of pressure and velocities. Adaptative mesh refinement is applied. The discrete equations are solved coupled by Newton iteration with Multigrid for linear system [2].

\subsection{F. Dabbene, CEA Saclay, France}

The flow model is an elliptic model based on an asymptotic analysis of the compressible Navier-Stokes equations at low Mach numbers. The system is solved by a semi-explicit Finite Element formulation using continuous bilinear test functions for momentum and discontinuous linear test functions on macro-elements for pressure, and implemented in the CEA code CAST3M [6]. Thermodynamic pressure is computed using the mass conservation constraint.

\subsection{A. Beccantini and E. Studer, CEA Saclay, France}

The equations solved are the Navier-Stokes equations for low-Mach number flows derived via asymptotic analysis and discretized using quadratic Finite Element and a semi-implicit projection method [1], also implemented in the CEA code CAST3M. Two different asymptotic models were considered, and the contributions will be denoted by "Beccantini" and "Studer" in the results section. In both cases, the thermodynamic pressure (constant in space) is computed by integrating the equation of state over the whole volume, under the mass conservation constraint.

\subsection{T. Kloczko, A. Beccantini and C. Corre, CEA Saclay, France and ENSAM/SINUMEF, Paris, France}

The full compressible Navier-Stokes equations are solved using an implicit Matrix-Free method coupled with a low-Mach number treatment (so-called low-Mach preconditioning) [5]. The explicit numerical flux is computed using an AUSM+ scheme extended to order 2 by MUSCL approach whereas the numerical flux in the implicit part is simply a Rusanov scheme of order 1 . The proposed approach exploits a particular property of a widely used low-Mach preconditioner proposed by Turkel.

\subsection{Heuveline, Heidelberg University, Germany}

Asymptotic equations are solved by a $h p$-Finite Element Method which allows the simultaneous adaptation of the mesh size $h$ and the polynomial degree $p$ of FEM ansatz in the context of a low-Mach number model. A duality-based a posteriori error analysis is developed for the conforming $h p$ Galerkin finite element approximation [4]. 
TABle 1. Test case $\mathrm{T} 1\left(\mathrm{Ra}=10^{6}, \epsilon=0.6\right.$ and constant properties, N.A. not available).

\begin{tabular}{|c|c|c|c|c|c|c|c|c|}
\cline { 2 - 9 } \multicolumn{1}{c|}{} & Vierendeels & Dabbene & Beccantini & Studer & Kloczko & Heuveline & Darbandi & $\begin{array}{c}\text { Reference } \\
\mathbf{2 0 0 0}\end{array}$ \\
\hline $\mathrm{Nu}(\mathrm{h})$ & $\mathbf{8 . 8 5 9 7 8}$ & 8.86380 & 8.85990 & 8.85980 & 8.86200 & $\mathbf{8 . 8 5 9 7 7 8}$ & 8.88000 & $\mathbf{8 . 8 5 9 7 8}$ \\
\hline $\mathrm{Nu}(\mathrm{c})$ & $\mathbf{8 . 8 5 9 7 8}$ & 8.86200 & 8.86007 & 8.85990 & 8.86380 & $\mathbf{8 . 8 5 9 7 8}$ & 8.88000 & $\mathbf{8 . 8 5 9 7 8}$ \\
\hline $\mathrm{Nu}(y=0.5)(\mathrm{h})$ & 7.81938 & 7.82170 & 7.81978 & N.A. & 7.82010 & N.A. & N.A. & N.A. \\
\hline $\mathrm{Nu}(y=0.5)(\mathrm{c})$ & 8.79636 & 8.81710 & 8.79646 & N.A. & 8.79750 & N.A. & N.A. & N.A. \\
\hline $\mathrm{Numax}(\mathrm{h})$ & 19.59642 & 19.62600 & 19.59538 & 19.59500 & 19.61070 & 19.59633 & N.A. & N.A. \\
\hline Numin(h) & $\mathbf{1 . 0 7 3 4 5}$ & 1.07690 & 1.07356 & 1.07360 & 1.07380 & $\mathbf{1 . 0 7 3 4 5}$ & N.A. & N.A. \\
\hline Numax $(\mathrm{c})$ & 16.36225 & 16.35200 & 16.36333 & 16.36100 & 16.37510 & 16.36226 & N.A. & N.A. \\
\hline Numin(c) & 0.85512 & 0.86102 & 0.85542 & 0.85500 & 0.85620 & 0.85513 & N.A. & N.A. \\
\hline Pmax/Po & $\mathbf{0 . 8 5 6 3 4 0}$ & 0.85661 & $\mathbf{0 . 8 5 6 3 4}$ & $\mathbf{0 . 8 5 6 3 4}$ & 0.85650 & $\mathbf{0 . 8 5 6 3 4}$ & 0.85500 & $\mathbf{0 . 8 5 6 3 3 8}$ \\
\hline Pmin/Po & $\mathbf{0 . 8 5 6 3 3 6}$ & 0.85661 & 0.85633 & $\mathbf{0 . 8 5 6 3 4}$ & 0.85650 & N.A. & N.A. & N.A. \\
\hline Type of & $2048 \times 2048$ & $320 \times 320$ & $296 \times 296$ & $80 \times 80$ & $240 \times 240$ & & $300 \times 300$ & N.A. \\
\hline Mesh & $4.2 \times 10^{6}$ & 102400 & 87616 & 6400 & 57600 & 200000 & & \\
\hline
\end{tabular}

\subsection{Darbandi and S.F. Hosseinizadeh, Sharif University of Technology, Teheran, Iran}

The authors introduce an easy two-steps modification scheme in order to include density variation in a specific incompressible algorithm. This scheme can be applied to other constant density algorithms. In this regard, the Navier-Stokes equation can be treated using a Finite-Volume approach [3]. (Note that this contribution was received in November 2004.)

\section{Conference Results (June 2004)}

In all cases, contributors were asked to produced grid-converged data or data on a series of sufficiently fine grids so that the Richardson extrapolation could be applied. In practice, some contributors provided the solutions they were able to compute (constraints of time, CPU, etc.). For the all three test-cases, we notice that asymptotic models and compressible models converge towards the reference solution found in January 2000. The latest solutions of Vierendeels in particular are for all three cases, T1, T2 and T3, very close to the reference solutions.

\section{List OF PITFALLS AND RECOMMENDATIONS}

The test case problem proposed is, at a first glance, a very easy problem to solve: steady two-dimensional laminar flow of a perfect gas in a very simple geometry. The boundary conditions are also very simple: no-slip condition and either imposed temperature or zero heat flux. However, as was experienced during the first worshop in 2000, and again during the 2004 benchmark, the problem is deceiptively simple, and obtaining the "correct" solution can be quite hard. Pitfalls to be avoided are:

- Mass conservation issue. Since the solutions sought are steady-state solutions of the Navier-Stokes equations, one may be tempted to accelerate the convergence to the steady state as is usually done in the case of external flows. However, because the flow is completely confined, one must guarantee that the mass of fluid in the cavity is conserved. In practice, one may impose at every time-step or iteration, that mass is conserved. In the low Mach number regime, this implies a rescaling of the thermodynamic pressure. 
TABle 2. Test case $\mathrm{T} 2\left(\mathrm{Ra}=10^{6}, \epsilon=0.6\right.$ and Sutherland law, N.A. not available).

\begin{tabular}{|c|c|c|c|c|c|c|c|c|}
\hline & Vierendeels & Braack & Dabbene & Beccantini & Kloczko & Heuveline & Darbandi & $\begin{array}{c}\text { Reference } \\
2000\end{array}$ \\
\hline $\mathrm{Nu}(\mathrm{h})$ & 8.6866 & 8.6866 & 8.6916 & 8.6868 & 8.6953 & 8.6889 & 8.7150 & 8.6866 \\
\hline $\mathrm{Nu}(\mathrm{c})$ & 8.6866 & 8.6866 & 8.6855 & 8.6747 & 8.6338 & 8.6861 & 8.7150 & 8.6866 \\
\hline $\mathrm{Nu}(y=0.5)(\mathrm{h})$ & 7.4593 & N.A. & 7.4552 & 7.4604 & 7.7 .4633 & N.A. & N.A. & N.A. \\
\hline $\mathrm{Nu}(y=0.5)(\mathrm{c})$ & 8.6372 & N.A. & 8.6587 & 8.6291 & 8.5892 & N.A. & N.A. & N.A. \\
\hline $\operatorname{Numax}(\mathrm{h})$ & 20.2704 & N.A. & 20.3100 & 20.2673 & 20.3219 & 20.3051 & N.A. & N.A. \\
\hline $\operatorname{Numin}(\mathrm{h})$ & 1.0667 & N.A. & 1.0738 & 1.0670 & 1.0668 & 1.0674 & N.A. & N.A. \\
\hline $\operatorname{Numax}(\mathrm{c})$ & 15.5194 & N.A. & 15.4690 & 15.4825 & 15.3805 & 15.5072 & N.A. & N.A. \\
\hline $\operatorname{Numin}(\mathrm{c})$ & 0.7575 & N.A. & 0.7604 & 0.7582 & 0.7598 & 0.7567 & N.A. & N.A. \\
\hline $\mathrm{Pmax} / \mathrm{Po}$ & 0.924489 & 0.924487 & 0.9255 & 0.9245 & 0.9248 & 0.9249 & 0.9225 & 0.924487 \\
\hline $\mathrm{Pmin} / \mathrm{Po}$ & 0.924485 & 0.924487 & 0.9255 & 0.9245 & 0.9248 & N.A. & N.A. & N.A. \\
\hline $\begin{array}{l}\text { Type of } \\
\text { Mesh }\end{array}$ & $\begin{array}{c}2048 \times 2048 \\
4.2 \times 10^{6}\end{array}$ & $\begin{array}{c}\text { locally refined, } \\
33784\end{array}$ & $\begin{array}{c}320 \times 320 \\
102400\end{array}$ & $\begin{array}{c}169 \times 148 \\
25012\end{array}$ & $\begin{array}{c}160 \times 160 \\
25600\end{array}$ & 200000 & $300 \times 300$ & N.A. \\
\hline
\end{tabular}

TABle 3. Test case T3 ( $\mathrm{Ra}=10^{7}, \epsilon=0.6$ and Sutherland law, N.A. not available).

\begin{tabular}{|c|c|c|c|c|c|c|}
\cline { 2 - 7 } & Vierendeels & Dabbene & Kloczko & Heuveline & Darbandi & $\begin{array}{c}\text { Reference } \\
\mathbf{2 0 0 0}\end{array}$ \\
\hline $\mathrm{Nu}(\mathrm{h})$ & $\mathbf{1 6 . 2 4 1 0}$ & 16.227 & 16.302 & 16.242 & 16.370 & $\mathbf{1 6 . 2 4 1 0}$ \\
\hline $\mathrm{Nu}(\mathrm{c})$ & $\mathbf{1 6 . 2 4 1 0}$ & 16.189 & 16.249 & 16.224 & 16.370 & $\mathbf{1 6 . 2 4 1 0}$ \\
\hline $\mathrm{Nu}(y=0.5)(\mathrm{h})$ & 13.189 & 12.850 & 13.180 & N.A. & N.A. & N.A. \\
\hline $\mathrm{Nu}(y=0.5)(\mathrm{c})$ & 15.512 & 15.265 & 15.376 & N.A. & N.A. & N.A. \\
\hline $\mathrm{Numax}(\mathrm{h})$ & 46.379 & 46.251 & 47.010 & 46.464 & N.A. & N.A. \\
\hline Numin(h) & 1.454 & 1.456 & 1.470 & 1.455 & N.A. & N.A. \\
\hline Numax(c) & 34.272 & 33.654 & 34.415 & 34.163 & N.A. & N.A. \\
\hline Numin(c) & 1.089 & 1.088 & 1.119 & 1.088 & N.A. & N.A. \\
\hline Pmax/Po & $\mathbf{0 . 9 2 2 6 4}$ & $\mathbf{0 . 9 2 3}$ & $\mathbf{0 . 9 2 3}$ & $\mathbf{0 . 9 2 3}$ & 0.918 & $\mathbf{0 . 9 2 2 6 3}$ \\
\hline Pmin/Po & $\mathbf{0 . 9 2 2 6 3}$ & $\mathbf{0 . 9 2 3}$ & $\mathbf{0 . 9 2 3}$ & N.A. & N.A. & N.A. \\
\hline $\begin{array}{c}\text { Type of } \\
\text { Mesh }\end{array}$ & $2048 \times 2048$ & $160 \times 160$ & $100 \times 100$ & & $300 \times 300$ & N.A. \\
\hline
\end{tabular}

- Calculations of the Nusselt number. The post-processing of the heat flux (for example by reconstructing gradients of temperature at the wall) should be consistent with the way the heat flux is approximated in the flow equation.

In order to check the quality of the solution, one must therefore check that at steady-state,

- the mass of fluid at steady state is equal to the initial mass of fluid (conservation of mass);

- the average Nusselt number on the left wall is equal to the average Nusselt number on the right wall (conservation of energy). 


\section{Conclusions}

A test case problem for low Mach number solvers was presented, dealing with natural convection flow subjected to large temperature differences. It was the object of a first international workshop in 2000, after which some reference (i.e. grid- and model-independent) solutions were produced, and the object of a second benchmark in the framework of the conference on "Mathematical and numerical aspects of low Mach number flows" organized by INRIA and MAB in June 2004. Different contributions for this conference were received, and have been reported in this paper. Some grid-independent solutions were also produced (using adaptive grid refinement or $h-p$ adaptive methods) which confirm the reference results of 2000 .

\section{REFERENCES}

[1] A. Beccantini et al., Simulation of Hydrogen Release and Combustion in large Scale Geometries: Models and Methods, Int. Conf. Supercomputing for Nuclear Applications (SNA 2003), Paris, 22-24 September, 2003.

[2] R. Becker and M. Braack, Multigrid techniques for finite elements on locally refined meshes. Numer. Linear Algebra Appl. 7 (2000) 363-379.

[3] M. Darbandi and S.F. Hosseinizadeh, General pressure-correction strategy to include density variation in incompressible algorithms. AIAA J. Thermophysics Heat Transfer 17 (2003) 372-380.

[4] V. Heuveline, On higher-order mixed FEM for low Mach number flows: application to a natural convection benchmark problem. Internat. J. Numer. Methods Fluids 41 (2003) 1339-1356.

[5] T. Kloczko, C. Corre and A. Beccantini, A Matrix-Free Implicit Method for Flows at all Speeds, Proc. ICCFD3 Conference, Toronto, Canada, 11-15 July, 2004.

[6] H. Paillère and J.P. Magnaud, A Finite Element Elliptic Flow Solver for Low-Mach Number Compressible Flows, in 10th Int. Conf. Finite Elements in Fluids, Tuscon, Az, USA, 5-8 January (1998).

[7] J. Vierendeels, K. Riemslagh and E. Dick, A Multigrid Semi-Implicit Line-Method for Viscous Incompressible and Low-Mach Number Flows on High Aspect Ratio Grids. J. Comput. Phys. 154 (1999) 310-341.

To access this journal online: www.edpsciences.org 